\title{
QCOF: New RPL Extension for QoS and Congestion-Aware in Low Power and Lossy Network
}

\author{
Yousra Ben Aissa ${ }^{1,2,3,4} \oplus^{a}$, Hanen Grichi ${ }^{5}$, Mohamed Khalgui ${ }^{1}{ }^{\mathrm{b}}{ }^{\mathrm{b}}$, Anis Koubaa ${ }^{6,7}$ \\ and Abdelmalik Bachir ${ }^{4}{ }^{\mathrm{c}}$ \\ ${ }^{1}$ School of Intelligence Science and Engineering, Jinan University (Zhuhai Campus), Zhuhai 519070, China \\ ${ }^{2}$ National Institute of Applied Sciences and Technology (INSAT), University of Carthage, Tunisia \\ ${ }^{3}$ Information Faculty of Mathematical Physical and Natural Sciences, University of Tunis El Manar, Tunis, Tunisia \\ ${ }^{4}$ Computer Science Department, University of Biskra, Algeria \\ ${ }^{5}$ LISI Laboratory, Tunisia Polytechnic School, INSAT Institute, University of Carthage, Tunis, Tunisia \\ ${ }^{6}$ Prince Sultan University, Saudi Arabia \\ ${ }^{7}$ CISTER/INESC TEC and ISEP-IPP, Porto, Portugal
}

\begin{abstract}
Keywords: RPL Objective Function, DODAG Construction, Low Power and Lossy Network (LLN), Congestion-aware, New DODAG Request Messages (NDR and NDR-Ack).

Abstract: Low power and lossy networks (LLNs) require a routing protocol under real-time and energy constraints, congestion aware and packet priority. Thus, Routing Protocol for Low power and lossy network (RPL) is recommended by Internet Engineering Task force (IETF) for LLN applications. In RPL, nodes select their optimal paths towards their preferred parents after meeting routing metrics that are injected in the objective function (OF). However, RPL did not impose any routing metric and left it open for implementation. In this paper, we propose a new RPL objective function which is based on the quality of service (QoS) and congestion-aware. In the case paths fail, we define new RPL control messages for enriching the network by adding more routing nodes. Extensive simulations show that QCOF achieves significant improvement in comparison with the existing objective functions, and appropriately satisfies real-time applications under QoS and network congestion.
\end{abstract}

\section{INTRODUCTION}

The evolution of Internet of Things (IoT) leads to a great revolution in network communication. In IoT, a large number of devices, objects, and computers are interconnected using various connecting technologies, that are provided in IoT's link layer with the IEEE 802.15.4, which is standardized for the low power and lossy networks (LLNs) (Al-Turjman, 2017). The routing in LLNs has become one of the most challenging issues, which is found in the network with limited energy resources, processing, and bandwidth such as wireless sensor networks (WSNs) (Kumar et al., 2018)(Naidji et al., 2018). WSNs become more and more attractive by their integration in a real world of interconnected objects through in-

\footnotetext{
a (iD https://orcid.org/0000-0001-9237-8083

b (iD) https://orcid.org/0000-0001-6311-3588

c (iD https://orcid.org/0000-0001-5160-9412
}

ternet (Zeinab and Elmustafa, 2017)(Hafidi. et al., 2019)(Hafidi et al., 2018). The performance of WSNs is affected by limiting processing and memory, limiting energy, losing packet, delay, and real-time data (Talebi et al., 2018). The limited energy associated with WSNs is a major bottleneck of WSN technologies. Therefore, we need a specific protocol for LLN's like RPL (Routing protocol for LLNs), which is standardized by IETF (Internet Engineering Task Force) in 2011 (Khallef et al., 2017)(Lakhdhar et al., 2018)(Gu et al., 2018).

RPL is capable of effective building routes, broadcasting routing information with a little overhead, and providing small response time, because routes are readily available (Gaddour and Koubâa, 2012)(Khalgui and Thramboulidis, 2008). Nowadays, RPL became the standard routing protocol for the majority of IoT applications based on LLN's, where, many companies adopted it as their underlying technology like ZigBee Alliance (Gaddour et al., 2015)(Khalgui et al.,

Ben Aissa, Y., Grichi, H., Khalgui, M., Koubaa, A. and Bachir, A 
2008). Nevertheless, RPL is still under development and several issues remain open for improvement, in particular, respect the quality of service (QoS), avoid congestion, and energy consumption.

Despite the huge number of the proposed protocols in literature, real-time communication (Khalgui et al., 2005), energy consumption (Ghribi et al., 2018) and congestion control remain one of the research challenges in LLNs (Khalgui et al., 2007)(Ramdani. et al., 2019)(Ramdani et al., 2018), where, i) realtime communication is subjected to packet loss, interference, unreliable data, missing deadline, particularly, for environmental monitoring applications that require reliable network performance and provide data timely and reliably (Wang et al., 2018)(Qin et al., 2012)(Khalgui et al., 2019), ii) energy consumption depends on many operations like communication, processing, etc, which increases when these operations increase, such in IoT the most of devices are battery operated, thus the energy consumption will be the network dominator (Wang et al., 2018), and iii) congestion occurs when the traffic load exceeds the available link capacity, buffet node capacity, contiguous or cascading failures, or the need of multihop forwarding (Al-Kashoash et al., 2017). These circumstances lead to increase packet loss ratio, increase latency, increase delay, low throughput, waste energy, increase retransmissions, and affect network reliability (Karoui et al., 2017)(Khalgui et al., 2007). To overcome these major limitations, we need to put these circumstances as criteria for network communication.

In this paper, we are interested in improving the QoS and avoiding congestion in LLNs such as WSNs. In RPL, the objective function is responsible of finding routing paths, which allows to select the best route according to predefined criteria. This route is selected after meeting link metrics that are recommended to be used in LLNs. However, in RPL the objective function OF0 is based on one metric which is rank (node positions). In spite of the existing RPL extensions, RPL is still open in research and needs more improvement (that we discuss in Section 2), which motivates us to design a new RPL extension to overcome the existing RPL extension limitations. Therefore, we propose a new RPL extension that supports multiple routing nodes (multiple DODAGS), by modifying RPL's objective function (OF) and add other options to avoid congestion, like time-feasibility, energy-feasibility, link quality/capacity, input/output data, packet priority. The proposed QoS and Congestion-Aware Objective Function (QCOF) allows RPL to avoid congestion after maintaining network feasibility in time and energy. such QCOF is implemented by using linear programming with the objective to maximize packet transmission rate according to their priority, while using the minimal DODAG roots (routing nodes) (we discuss this idea in Section 4.2). In the case where all paths fail, we propose new RPL control messages NDR and NDR-Ack (see Sec. 4.2.1) to add a new DODAG while maintaining network feasibility.

The main originality in this paper are summarized as follows:

- Delivering packets according to theirs priorities,

- Using link capacity/quality and total input/output to detect, alleviate and avoid congestion,

- Guaranteeing network feasibility in time and energy,

- Using multiple DODAGs whenever and wherever they are needed,

- Using new RPL control message structure.

The rest of paper is organized as follows: Section 2 presents an overview about the routing protocol RPL. Section 3 summarizes related works. Section 4 provides a formal model and description for the proposed objective function. Section 5 evaluates the performance of the proposed solutions with a case study. Finally Section 6 provides concluding remarks and directions for a future work.

\section{RPL OVERVIEW}

RPL is a proactive routing protocol for LLNs as defined in RFC6550 (Request for Comments) (Shelby et al., 2012), based on distance vectors and operate on IEEE 802.15.4 (Molisch et al., 2004), which is standardized for constrained and IP-based environment, such as 6LoWPAN networks (IPv6 Low power Wireless Personal Area Networks), and it is known as the standard routing protocol for IoT based LLNs such as WSNs (Gaddour and Koubâa, 2012). In RPL, the network topology organized as DAG (Directed Acyclic Graph), which is similar to the tree, while in DAG nodes can associate to multiple parents not like tree. Specifically, nodes are organized as DODAGs (Destination Oriented DAGs) (Winter et al., 2012), where RPL assigned for each node in the network a rank, which represents the individual position of that node (Ghaleb et al., 2018). In fact, it increases monotonically while moving away from the root nodes (sink nodes or DODAG root) towards the leaf nodes, then inversely decreases from root nodes to leaf nodes. Whilst, data is transmitted upward to root nodes or downward to leaf nodes (Thubert, 2012).

The forwarding network topology built by RPL 
called DODAG, where each node identifies a set of available parents on a path towards the DODAG root (sink node), then selects one of them as the preferred parent based on the objective function. Thereafter, if a link between a node and its selected parent fails, then it switches to another parent from its available parents set. The objective function defines how RPL nodes would choose their preferred parents according to one or more metrics. However, the OF0 of RPL based only on one metric which is a rank (Thubert, 2012).

RPL offers a set of control messages that help nodes to choose their preferred parents, where nodes announce their ranks by sending a control messages called DODAG information object (DIO). After receiving DIOs, they start establishing their path towards their parent (DODAG root). Thereafter, they update their rank by rank sum of their preferred parents and the cost to reach them, to update their information throughout the DAG, nodes send DAO message (object of update to the destination). To get information about the network, nodes can send DIS (information request DODAG) messages for discovering existing networks (Ghaleb et al., 2018).

\section{RELATED WORK}

Most of research have been carried out improving RPL's objective function by adding several metrics. However, RPL's specification did not impose any routing metric and left it open for research.

The default RPL's objective function (OF0) is proposed as the first one in (Thubert, 2012). In OF0, the node always chooses the preferred parent according to the minimum rank. OF0 is a simple function which does not consider any routing metric. After OF0, the work reported in (Gnawali and Levis, 2012) proposes a new objective function called the minimum rank with hysteresis objective function (MRHOF), which works on adding metrics along the route. However, these two objective functions did meet all LLN's application requirements. The work reported in (Kim et al., 2017a) modifies RPL implementation to support diverse traffic patterns, termed DT-RPL, which updates link quality by using both upward and downward traffic. However, this work is based only on link quality which is not sufficient to satisfy LLN's application requirements. And in (Wang and Chalhoub, 2019), the authors proposes an enhancement mechanism for RPL based on a combination of multiple sinks support, RSSI, Rank and dynamic control message management. Despite, it uses more metrics which sill not sufficient to meet neither real-time
LLN's application requirements nor packet priority. Also, the work reported in (Wang et al., 2016) proposes a network life cycle index LCI to improve the original RPL. The index takes various factors into consideration, for instance, link quality, node energy, energy consumption rate, throughput and data rate. In (Lamaazi and Benamar, 2017), the authors proposes a new objective function based on fuzzy logic called OF-EC, which considers expected transmission count (ETX), hop count and energy consumption. However, these two works do not guarantee any respect for real-time constraint or packet priority. In (Gaddour et al., 2014), the authors design a new objective function based on fuzzy logic, which is called OFFL. This function combines four routing metrics to provide a routing decision toward parents. However, these works do not consider neither packet priority nor congestion.

The following research works deal with congestion-aware to improve RPL's objective function, where the work in (Al-Kashoash et al., 2016) proposes a new RPL's objective function called Congestion-Aware Objective Function (CA-OF), which uses buffer occupancy to minimize lost packets due to congestion. The work in (Kim et al., 2017b) studies the load balancing and congestion problem of RPL. It attempts to improve the end-to-end packet delivery performance by balancing the traffic load within a routing tree. The work in (Lodhi et al., 2015) proposes a multi-path RPL's extension (MRPL) which aims to provide temporary multiple routing paths during congestion over a path. However, these research works still not sufficient to meet real-time LLN's application requirements.

In the above-mentioned researches, the objective function based on one, two, or three metrics combination, which is not sufficient to satisfy all real-time LLN's application requirements, also, using two or three metrics may improve DAG performance according to the chosen metrics, but may lead to degradation according to other ones. Thus, in this paper we propose a new RPL's extension which addresses the limitation of the related works, by using new metrics combination and improve DODAG construction according to satisfy the real-time LLN's application requirements.

\section{QCOF: QoS AND CONGESTION-AWARE OBJECTIVE FUNCTION}

We propose a new objective function for RPL, which chooses the optimal feasible path to forward data from 
the source node to the root node (DODAG root). According to RPL, each node needs to select a preferred parent from its neighborhood (next-hop) based on the objective function. Thus, in the proposed QoS and Congestion-Aware Objective Function QCOF (see Sec. 4.2), the optimal feasible path is chosen after verifying the following constraints: 1) meets realtime constraints (time-feasibility); 2) meets energy constraints (energy-feasibility); 3) respects link capacity and total input/output data (congestion-aware); 4) sends data according to their priority. In the case where all paths fail, RPL attempts to add a new DODAG root by using a new control message types NDR and NDR-Ack (see Sec. 4.2.1).

\subsection{Notations}

This section formalises node characteristics and routing metrics used to design QCOF. Let $\mathcal{N}$ be the set of sensor nodes, and $n_{i}$ be a sensor node from $\mathcal{N}$, which has $\mathcal{M}_{s}^{j}$ a set of new periodic messages to send through channel $j$ over a link, and $\mathcal{M} O_{s}^{j}\left(\right.$ resp. $\left.\mathcal{M}_{r}^{j}\right)$ a set of old periodic messages that are transmitted (resp. receive) through channel $j$. In fact, a sensor node has a set of channels $\mathcal{C}$ over one link.

- Definition 1: A link $(i, k)$ exists between two nodes $n_{i}$ and $n_{k}$, or $n_{k}$ is the neighbor of $n_{k}$, if $n_{k}$ is the communication range of $n_{i}\left(R_{i}\right)$. Let $N_{i}$ be the set of neighbor nodes of $n_{i}$, which is given by

$$
N_{i}=\left\{(i, k) / \text { dist }_{i, k} \leq R_{i}, i, k \in \mathcal{N}\right\}
$$

where, dist $t_{i, k}$ is the distance between nodes $n_{i}$ and $n_{k}$.

- Definition 2: Node $n_{i}$ can forward its data to node $n_{k}$ if

$$
\begin{aligned}
F n_{i, k}= & \left\{k \in N_{i} / \text { dist }_{k, \text { sink }}<\text { dist }_{i, \text { sink }}\right. \\
& \left.\& \operatorname{Rank}\left(\mathrm{n}_{\mathrm{k}}\right)<\operatorname{Rank}\left(\mathrm{n}_{\mathrm{i}}\right)\right\}
\end{aligned}
$$

- Definition 3: As defined in (Aissa et al., 2019) and (Aissa et al., 2018), Real-time data can be sent over channel $j$ if and only if

- Channel utilization $\left(U_{j}\right)$ is less than 1, i.e.,

$$
U_{j}=\sum_{i=1}^{\left|\mathcal{M}_{s}^{j}\right|} \frac{W C T T_{i, j}}{T_{i}} \leq 1
$$

- Consumed energy by this channel $\left(E_{j}\right)$ is less than available energy, i.e.,

$$
\sum_{j=1}^{|C|} E_{j}\left(\left[t_{1}, t_{2}\right]\right)<C_{B}\left(t_{1}\right)+E_{H}\left(\left[t_{1}, t_{2}\right]\right)
$$

where $W C T T_{i, j}$ is worst case transmission time of message $i$ over channel $j, E_{j}\left(\left[t_{1}, t_{2}\right]\right)$ is consumed energy by channel $j$ in time interval $\left[t_{1}, t_{2}\right] . C_{B}\left(t_{1}\right)$ is remained energy in battery at $t_{1}$, and $E_{H}\left(\left[t_{1}, t_{2}\right]\right)$ is harvested energy which is collected from a photovoltaic (PV) power source $P_{\mathrm{PV}}(t)$ at particular time $t$. The harvested energy in time interval $\left[t_{1}, t_{2}\right]$ is given by

$$
E_{H}\left(\left[t_{1}, t_{2}\right]\right)=\int_{t=t_{1}}^{t_{2}} P_{\mathrm{PV}}(t) d t
$$

- Definition 4:A link between nodes $n_{i}$ and $n_{k}$ may have congestion in the future, if

- the sum of received/transmitted (input/output) messages is bigger than the predefined threshold (exp. threshold $=0.8$ ), i.e.,

$$
\begin{aligned}
& \forall c_{j} \in \mathcal{C}: \\
& L U_{i, k}=\sum_{k=1}^{\left|\mathcal{M}_{r}^{j}\right|} \frac{W C R T_{k, j}}{T_{k}}+ \\
& \sum_{i=1}^{\left|\mathcal{M}_{s}^{j}+\mathcal{M} O_{s}^{j}\right|} \frac{W C T T_{i, j}}{T_{i}}>\text { Threshold }
\end{aligned}
$$

- the set of received messages is bigger than the set of transmitted messages, i.e.,

$$
\begin{aligned}
& \forall c_{j} \in \mathcal{C}: \\
& \sum_{k=1}^{\left|\mathcal{M}_{r}^{j}\right|} \frac{W C R T_{k, j}}{T_{k}}>\sum_{i=1}^{\left|\mathcal{M}_{s}^{j}+\mathcal{M} O_{s}^{j}\right|} \frac{W C T T_{i, j}}{T_{i}}
\end{aligned}
$$

where $W C R T_{k, j}$ (res. $W C T T_{i, j}$ ) is worst case reception time of message $k$ from node $n_{k}$ (res. transmission time of message $i$ from node $n_{i}$ ) over channel $j$, and $T_{k}$ (res. $T_{i}$ ) refers to the period of message $k$ (res. message $i$ ).

- Definition 5: Let us suppose that the priority of a packet varies between 1 to 10 . Thus, node $n_{i}$ has higher priority $\left(P_{i}\right)$ than node $n_{k}$, if and only if

$$
P_{i}>P_{k}, \text { where } P_{i} \text { and } P_{k} \in[1-10]
$$

\subsection{Objective Function}

In this paper, we propose a new RPL's objective function which is based on Quality of Service and Congestion-aware QCOF. QCOF uses the combination of routing metrics and constraints to choose the feasible path, i.e., the rank (node position) (as defined in (Thubert, 2012)), real-time constraints, energy constraints, packet priority, congestion metrics. In RPL, each node uses the objective function to choose its preferred parent, which is used to transfer data towards the DODAG root. Thus, each node uses QCOF to establish its path to the DODAG root. 
QCOF is implemented by using linear programming, where the objective function is to maximize packet transmission rate according to their priority (Eq. 9.0), which is subjected to five constraints, given by: i) for each channel between node $n_{i}$ and its parent $n_{j}$; the channel's utilization over their link $F n_{i, j}$ must not exceed the predefined threshold (Eq. 9.1), ii) for each node; its output data (transmitting data) must be higher than the input data (receiving data) (Eq. 9.2), iii) for each channel; its consumed energy must not exceed the available energy for a node (Eq. 9.3), vi) for each data; its coefficient's value must be between one and zero, for identifying if the data will be sent or not (Eq. 9.4), v) for each data; its coefficient's sum $\alpha_{i, j}$ must equal to 1 , for guaranteeing that the transmission will not be duplicated (Eq. 9.5).

$$
\left\{\begin{aligned}
\text { Maximize } & \sum_{j=1}^{|\mathcal{C}|} \sum_{i=1}^{\left|\mathcal{M}_{s}^{j}\right|} \alpha_{i, j} P_{i} \quad(9.0) \\
\text { Subject to } & \forall n_{j} \in F n_{i, j}, \forall c_{j} \in \mathcal{C}: \\
& \sum_{i=1}^{\left|\mathcal{M}_{s}^{j}\right|} \frac{\alpha_{i, j} W C T T_{i, j}}{T_{i}}+\sum_{i=1}^{\left|\mathcal{M} O_{s}^{j}\right|} \frac{W C T T_{i, j}}{T_{i}} \\
& +\sum_{k=1}^{\left|\mathcal{M}_{r}^{j}\right|} \frac{W C R T_{k, j}}{T_{k}}+\leq \text { Threshold } \\
& \left|\mathcal{M}_{r}^{j}\right| \\
& \sum_{k=1}^{\mid \mathcal{M}^{j}} \frac{W C R T_{k, j}}{T_{k} \mid} \leq \sum_{i=1}^{\left|\mathcal{M}_{s}^{j}\right|} \frac{\alpha_{i, j} W C T T_{i, j}}{T_{i}} \\
& +\sum_{i=1} \frac{(9.1)}{T_{i}} \\
& |\mathcal{C}| \\
& \sum_{j=1} E_{j}\left(\left[t_{1}, t_{2}\right]\right)<C_{B}\left(t_{1}\right)+E_{H}\left(\left[t_{1}, t_{2}\right]\right) \\
& \forall m_{i} \in \mathcal{M}_{s}, 0 \leq \alpha_{i, j} \leq 1 \\
& \forall m_{i} \in \mathcal{M}_{s}, \sum_{j=1}^{|C|} \alpha_{i, j}=1
\end{aligned}\right.
$$

\subsubsection{DODAG Construction}

RPL's objective function is defined to construct the DODAG, but in case unsatisfying constraints, the OF cannot construct the DODAG, i.e., the data transmission will not respect the objective function's constrains, which can lead to disaster. In WSN root nodes are high cost and energy that offered with a limited number. Thus, we suggest to manage DODAG root's locations. Therefore, initially the WSN has only one DODAG root, then it starts adding more DODAG roots one by one, whenever and wherever they are needed based on QCOF. Hence, RPL attempts to place multiple DODAGs in the highly loaded areas according to QCOF constraints. Whereas, the process of enriching the network by adding more DODAG roots based on RPL's control messages. For constructing DODAG RPL has four control message's types: 1) DODAG Information Object (DIO) used to create path from upward routing, 2) Destination Advertisement Object(DAO) used to create path from downward routing, also to propagate destination information to the upward nodes, 3) DODAG Information Solicitation (DIS) used to solicit or request a DIO from the RPL node, also to search neighborhood, 4) Destination Advertisement Object Acknowledgment (DAO-ACK) is response to unicast DAO message. However, these control messages cannot use in this process (enriching the network), thus, we define new RPL's control messages which is a New DODAG Request message (NDR) and New DODAG Acknowledgment message (NDR-Ack). NDR message requests to assign a new DODAG root to a node, and NDR-Ack message is the response to the multicast NDR message. NDR (resp. NDR-Ack) structure is based on DODAG Repair Request (resp. Reply) (DRQ , resp. DRP) message's structure (see (Guo et al., 2013)), the structure of NDR and NDR-Ack message is shown in Fig. 1 and Fig. 2) respectively.

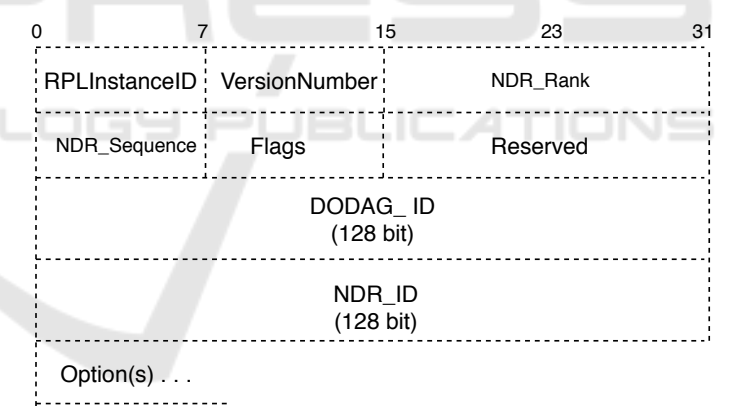

Figure 1: NDR message structure.

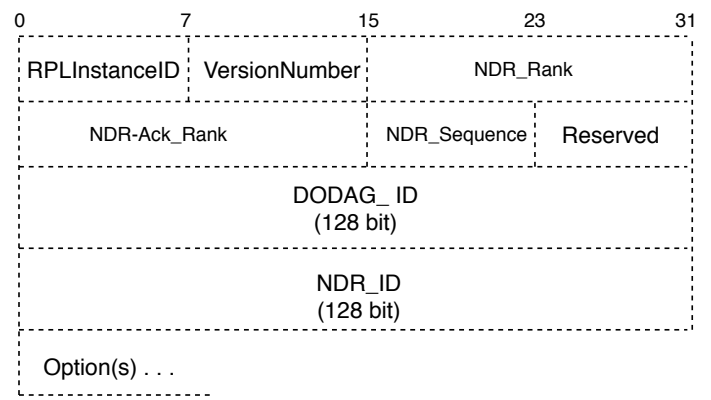

Figure 2: NDR-Ack message structure. 
Where,

- RPLInstanceID: is unsigned field used to indicate the part of RPL instance in the DODAG, as described in (Winter et al., 2012).

- Version Number: is unsigned integer to indicate DODAG version's number as described in (Winter et al., 2012).

- NDR_Rank: is unsigned integer with 16-bit used to indicate the rank (position) of the node generating the NDR message.

- NDR-Ack_Rank: is unsigned integer with 16-bit to indicate the rank (position) of the DODAG root sending the NDR-Ack message.

- NDR_Sequence: is a field with 8-bit to indicate the sequence number of NDR message at the node generating the NDR message.

- Flags: is unused field with 8-bit, which is reserved for flags. The field MUST be initialized to zero by sender and MUST be ignored by receiver as defined in (Winter et al., 2012).

- Reserved: is unused field, which MUST be initialized to zero by sender and MUST be ignored by receiver as defined in (Winter et al., 2012).

- DODAG_ID: is a field with 128-bit, which identifies the DODAG root. It must be a routable IPv6 address belonging to the DODAG root as defined in (Winter et al., 2012).

- NDR_ID: is an IPv6 address (128-bit) of the node generating NDR message.

A node which could not satisfies QCOF constraints broadcasts a NDR message to the no-assigned DODAG roots. Then, a DODAG root which is near to the sender node replies by NDR-Ack message. If the node received NDR-Ack, then it adds the address of the DODAG root to its preferred parent list, and starts transmitting data through this DODAG root (See Fig. 3 ). This process stays usable while there is available DODAG root.

\section{SIMULATIONS}

In order to evaluate the impact of the proposed RPL's objective function QCOF on QoS and congestion, we consider a DAG which contains up to one hundred nodes, that are spread randomly in order to form a connected network. These nodes have to send five hundred new random packets to the DODAG root. To show how QCOF can provide good performance with respect real-time application requirements, we compare QCOF with the objective function OF0 and

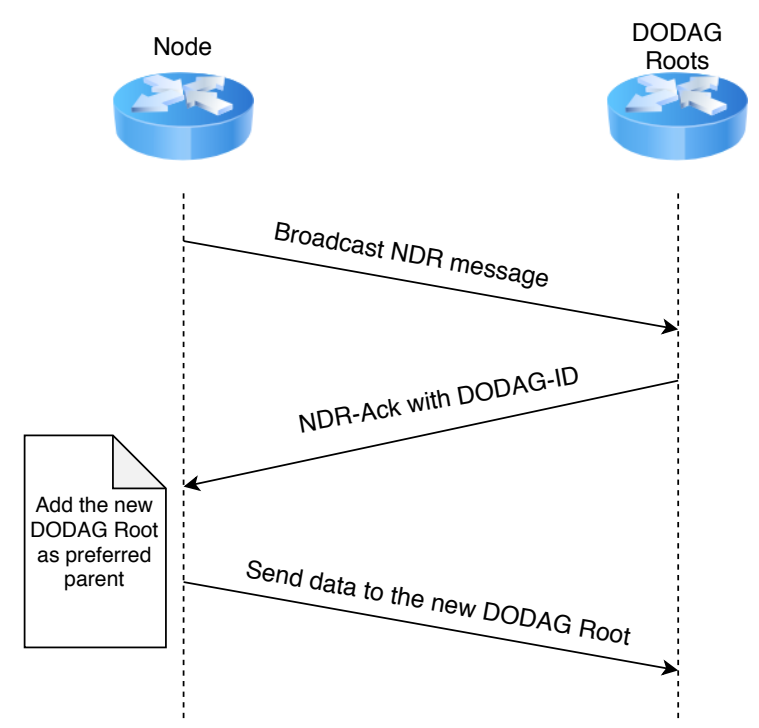

Figure 3: DODAG construction.

MRHOF, that are simulated in Contiki ${ }^{1}$, which is an open source operating system for the IoT. Then, we measure five performance metrics while ensuring that the network is time-feasible and energy-feasible. For modeling and solving the optimization problem defined in QCOF, we use CPLEX tool ${ }^{2}$.

\subsection{Performance Metrics}

- Packet Delivery Ratio: is the ratio of packets successfully received by the destinations to the total sent by the sources, which can be defined as following

$$
P D R=\frac{\operatorname{Data}_{R}}{\operatorname{Data}_{S}}
$$

Where, $\operatorname{Data}_{R}$ is the total successfully received packets, and Data $a_{S}$ is the total sent packets.

- Priority Packet Delivery Ratio: is the ratio of packets delivery according to their priorities to the total priorities.

- Throughput: is the total delivered packets over the total simulation time.

- Average Energy Consumption: is the total consumed energy by each packet over the total sent and received packets.

\subsection{Simulation Results}

Packet Delivery Ratio. Figure 4 shows the packet delivery ratio after varying the network size. As

\footnotetext{
${ }^{1}$ http://www.contiki-os.org/

${ }^{2} \mathrm{https}: / /$ www.ibm.com/analytics/cplex-optimizer
} 


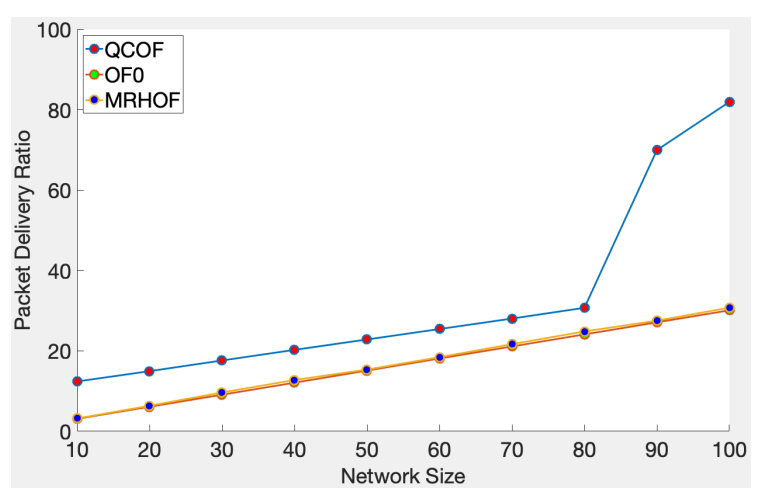

Figure 4: Packet delivery ratio versus packet priorities.

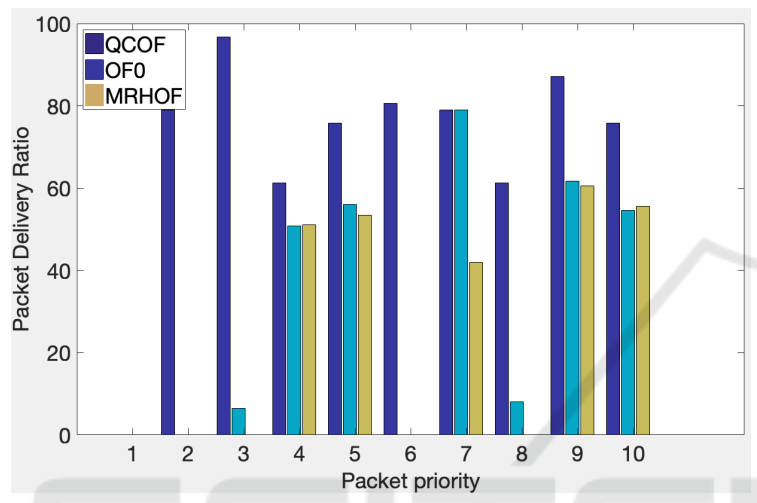

Figure 5: Packet delivery ratio versus network size.

shown in this figure and based on our simulation statistics, the proposed objective function QCOF always provides the highest packets delivery ratios compared to the related works OF0 and MRHOF, where it goes up $80 \%$ when the network size goes to 100 nodes. Whereas OF0 and MRHOF provide approximately same ratio.

Priority Packet Delivery Ratio. Figure 5 shows the packet delivery ratio according to their priorities, where a set of periodic packets is coming with the same WCTT and random period. Packet priorities is between 1 and 10, and packets are delivered sequentially according to their arrival time. As shown in this figure and based on our simulation statistics, the proposed objective function QCOF always provides the highest ratios compared to the related works OF0 and MRHOF, that is because QCOF provides the highest packet delivery ratios.

Throughput. Figure 6 shows the throughput computation after varying the network size. As shown in this figure and based on our simulation statistics, QCOF always produces the highest throughput compared with the existing related work OF0 and MRHOF, also, we noticed that the throughput is di-

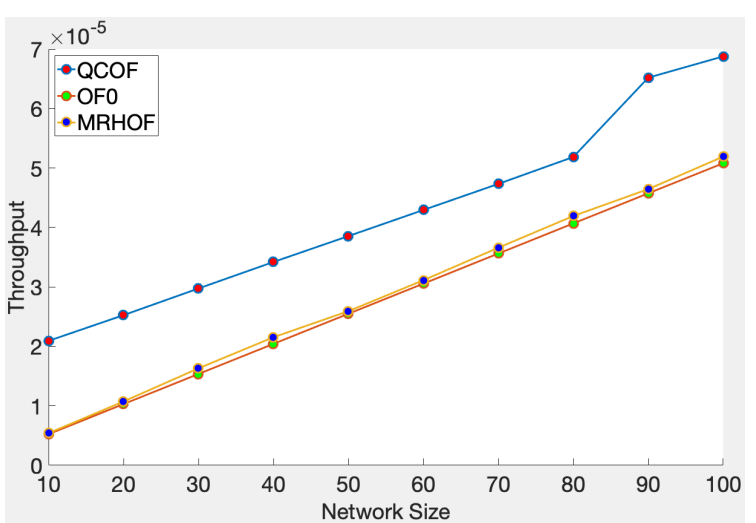

Figure 6: Throughput versus network size.

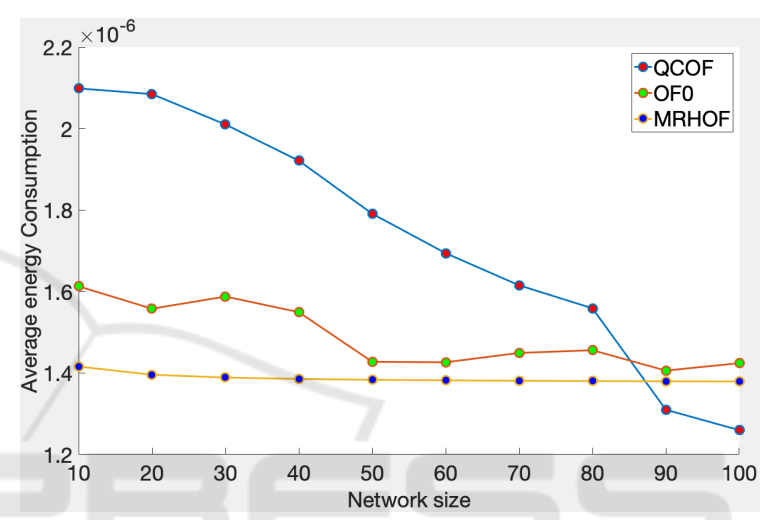

Figure 7: Average energy consumption versus network size.

rectly proportional to the packet delivery ratio, which represents the quality of network connection, where when the throughput is increased, the network connection's quality becomes better and better.

Average Energy Consumption. To evaluate the effect of the proposed RPL's objective function QCOF on the consumed energy, we run an extensive simulation and measure the average energy consumption of each objective functions, where we use energy harvesting and PowerControl as defined in (Aissa et al., 2019). Then we plot the obtained results in Fig. 7. As shown in this figure, QCOF achieves the highest average when the number of sensor node is less than 90 nodes, whereas, it provides the lowest one as much as network size increases, such that packet delivery ratio increases as much as sensor nodes number increases. While the average provided by $\mathrm{OF} 0$ is not continuous decreases/increases to be the highest one when network size passed over 90 nodes, that is due to packet loss. MRHOF provides the lowest average which did not affect by network size, i.e., the provided packet delivery ratio is strict whatever network size increases or decreases. 


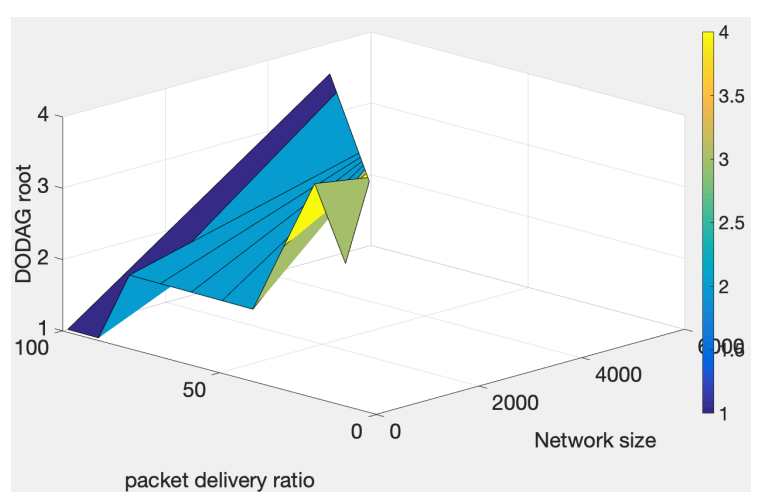

Figure 8: DODAG root numbers versus network size and packet delivery ratio.

DODAG Roots Number. Figure 8 shows the variation of using DODAG roots versus network size and packet delivery ratio. As shown in this figure, DODAG root number is proportional to the packet delivery ratio, where based on our simulation statistics, for high packet delivery ratio, the network adds more DODAG roots to meet the real-time application requirements. Additionally and according to our simulation statistics, DODAG roots number does not have directed proportional relation to network size, as shown in this figure, for a network with 10 nodes the number of used DODAG roots is three node, and for 20 nodes it uses four DODAG roots, then for 100 nodes it uses one DODAG root. That is because the high packets delivery ratio compared to OF0 and MARHOF.

Our experiments show that the proposed objective function QCOF provides the best result compared to the existing works OF0 and MRHOF, according to the measured performance metrics: throughput, packet delivery ratio, packet delivery ratio according to their priority, average energy consumption.

\section{CONCLUSIONS}

In this paper, we have proposed a new RPL's objective function QCOF, which attempts to avoid congestion in order to respect real-time application requirements, while sensor nodes can perform energy harvesting and use PowerControl. After comparing QCOF with the existing objective functions that use only one, two or three metrics that are not sufficient to meet the application requirements, we found that QCOF is more appropriate to respect real-time application requirements, where QCOF combines five metrics, such as node rank, real-time constraints, energy constraints, link capacity (Threshold), and Input/Output. QCOF is not like other related existing objective functions, where it sends packets according to their priorities, and in case paths fail, nodes can ask for new DODAG roots, that are assigned according to their availability and node positions. In fact, we propose new RPL's control messages, i.e., NDR and NDR-Ack. NDR sends from the node which announced path fails to a set of DODAG roots, if there is an available DODAG root near to that node, then it replies by NDR-Ack. Once the node receives the NDR-Ack, it adds this DODAG root as a preferred parent and starts forwarding data.

Extensive simulation experiments show that the proposed objective function achieves a significant improvement over the related works, where it achieves the highest packets delivery ratio, in particular, packets delivery ratio according to packet priorities, and the best throughput compared with a low average in the consumed energy. As future work, we plan to implement QCOF in practice on real case study.

\section{REFERENCES}

Aissa, Y. B., Bachir, A., Khalgui, M., Koubaa, A., Li, Z., and $\mathrm{Qu}, \mathrm{T}$. (2019). On feasibility of multichannel reconfigurable wireless sensor networks under real-time and energy constraints. IEEE Transactions on Systems, Man, and Cybernetics: Systems, pages 1-16.

Aissa, Y. B., Mosbahi, O., Khalgui, M., and Bachir, A. (2018). New scheduling mechanism in multi-channel reconfigurable wsn under qos and energy constraints. In 32nd annual European Simulation and Modelling Conference 2018, pages 187-191, Ghent, Belgium.

Al-Kashoash, H. A., Al-Nidawi, Y., and Kemp, A. H. (2016). Congestion-aware rpl for 610wpan networks. In 2016 Wireless Telecommunications Symposium (WTS), pages 1-6. IEEE.

Al-Kashoash, H. A., Hafeez, M., and Kemp, A. H. (2017). Congestion control for 6lowpan networks: A game theoretic framework. IEEE internet of things journal, 4(3):760-771.

Al-Turjman, F. M. (2017). Information-centric sensor networks for cognitive iot: an overview. Annals of Telecommunications, 72(1-2):3-18.

Gaddour, O. and Koubâa, A. (2012). Rpl in a nutshell: A survey. Computer Networks, 56(14):3163-3178.

Gaddour, O., Koubâa, A., and Abid, M. (2015). Quality-ofservice aware routing for static and mobile ipv6-based low-power and lossy sensor networks using rpl. Ad Hoc Networks, 33:233-256.

Gaddour, O., Koubâa, A., Baccour, N., and Abid, M. (2014). Of-fl: Qos-aware fuzzy logic objective function for the rpl routing protocol. In 2014 12th International Symposium on Modeling and Optimization in Mobile, Ad Hoc, and Wireless Networks (WiOpt), pages 365-372. IEEE.

Ghaleb, B., Al-Dubai, A., Ekonomou, E., Alsarhan, A., Nasser, Y., Mackenzie, L., and Boukerche, A. (2018). 
A survey of limitations and enhancements of the ipv6 routing protocol for low-power and lossy networks: A focus on core operations. IEEE Communications Surveys \& Tutorials.

Ghribi, I., Abdallah, R. B., Khalgui, M., Li, Z., Alnowibet, K., and Platzner, M. (2018). R-codesign: Codesign methodology for real-time reconfigurable embedded systems under energy constraints. IEEE Access, 6:14078-14092.

Gnawali, O. and Levis, P. (2012). The minimum rank with hysteresis objective function. Technical report.

Gu, C., Li, Z., Wu, N., Khalgui, M., Qu, T., and Al-Ahmari, A. (2018). Improved multi-step look-ahead control policies for automated manufacturing systems. IEEE Access, 6:68824-68838.

Guo, J., Orlik, P., and Bhatti, G. (2013). Loop free dodag local repair. draft-guo-roll-loop-free-dodag-repair-01.

Hafidi, Y., Kahloul, L., Khalgui, M., Li, Z., Alnowibet, K., and $\mathrm{Qu}, \mathrm{T}$. (2018). On methodology for the verification of reconfigurable timed net condition/event systems. IEEE Transactions on Systems, Man, and Cybernetics: Systems, (99):1-15.

Hafidi., Y., Kahloul., L., Khalgui., M., and Ramdani., M. (2019). On improved verification of reconfigurable real-time systems. In Proceedings of the 14th International Conference on Evaluation of Novel Approaches to Software Engineering - Volume 1: ENASE, pages 394-401. INSTICC, SciTePress.

Karoui, O., Khalgui, M., Koubâa, A., Guerfala, E., Li, Z., and Tovar, E. (2017). Dual mode for vehicular platoon safety: Simulation and formal verification. Information Sciences, 402:216-232.

Khalgui, M., Carpanzano, E., and Hanisch, H.-M. (2008)

An optimised simulation of component-based embedded systems in manufacturing industry. International Journal of Simulation and Process Modelling, 4(2):148-162.

Khalgui, M., Mosbahi, O., and Li, Z. (2019). On reconfiguration theory of discrete-event systems: From initial specification until final deployment. IEEE Access, 7:18219-18233.

Khalgui, M., Rebeuf, X., and Simonot-Lion, F. (2005). A schedulability analysis of an iec-61499 control application. IFAC Proceedings Volumes, 38(2):71-78.

Khalgui, M., Rebeuf, X., and Simonot-Lion, F. (2007). A deployment method of component based applications on distributed industrial control systems. European Jounal of Automated Systems, 41(6):707-732.

Khalgui, M. and Thramboulidis, K. (2008). An iec61499based development approach for distributed industrial control applications. International Journal of Modelling Identification and Control, 4(2):186.

Khallef, W., Molnar, M., Benslimane, A., and Durand, S. (2017). Multiple constrained qos routing with rpl. In 2017 IEEE International Conference on Communications (ICC), pages 1-6. IEEE.

Kim, H.-S., Cho, H., Kim, H., and Bahk, S. (2017a). Dt-rpl: Diverse bidirectional traffic delivery through rpl routing protocol in low power and lossy networks. Computer Networks, 126:150-161.
Kim, H.-S., Kim, H., Paek, J., and Bahk, S. (2017b). Load balancing under heavy traffic in rpl routing protocol for low power and lossy networks. IEEE Transactions on Mobile Computing, 16(4):964-979.

Kumar, A., Zhao, M., Wong, K.-J., Guan, Y. L., and Chong, P. H. J. (2018). A comprehensive study of iot and wsn mac protocols: Research issues, challenges and opportunities. IEEE Access, 6:76228-76262.

Lakhdhar, W., Mzid, R., Khalgui, M., Li, Z., Frey, G., and Al-Ahmari, A. (2018). Multiobjective optimization approach for a portable development of reconfigurable real-time systems: From specification to implementation. IEEE Transactions on Systems, Man, and Cybernetics: Systems, (99):1-15.

Lamaazi, H. and Benamar, N. (2017). Rpl enhancement using a new objective function based on combined metrics. In 2017 13th International Wireless Communications and Mobile Computing Conference (IWCMC), pages 1459-1464. IEEE.

Lodhi, M. A., Rehman, A., Khan, M. M., and Hussain, F. B. (2015). Multiple path rpl for low power lossy networks. In 2015 IEEE Asia Pacific Conference on Wireless and Mobile (APWiMob), pages 279-284. IEEE.

Molisch, A. F., Balakrishnan, K., Chong, C.-C., Emami, S., Fort, A., Karedal, J., Kunisch, J., Schantz, H., Schuster, U., and Siwiak, K. (2004). Ieee 802.15. 4a channel model-final report. IEEE P802, 15(04):0662.

Naidji, I., Smida, M. B., Khalgui, M., and Bachir, A. (2018). Non cooperative game theoretic approach for residential energy management in smart grid. In The 32nd Annual European Simulation and Modelling Conference, pages 164-170, Ghent, Belgium.

Qin, M., Li, Z., Zhou, M., Khalgui, M., and Mosbahi, O. (2012). Deadlock prevention for a class of petri nets with uncontrollable and unobservable transitions. IEEE Transactions on Systems, Man, and Cybernetics-Part A: Systems and Humans, 42(3):727_ 738 .

Ramdani, M., Kahloul, L., and Khalgui, M. (2018). Automatic properties classification approach for guiding the verification of complex reconfigurable systems. In Proceedings of the 13th International Conference on Software Technologies - Volume 1: ICSOFT, pages 591-598. INSTICC, SciTePress.

Ramdani., M., Kahloul., L., Khalgui., M., and Hafidi., Y. (2019). R-TNCES rebuilding: A new method of ctl model update for reconfigurable systems. In Proceedings of the 14th International Conference on Evaluation of Novel Approaches to Software Engineering - Volume 1: ENASE, pages 159-168. INSTICC, SciTePress.

Shelby, Z., Chakrabarti, S., Nordmark, E., and Bormann, C. (2012). Neighbor discovery optimization for ipv6 over low-power wireless personal area networks (6lowpans). Technical report.

Talebi, M., Papatsimpa, C., and Linnartz, J.-P. M. (2018). Dynamic performance analysis of ieee 802.15. 4 networks under intermittent wi-fi interference. In 2018 IEEE 29th Annual International Symposium on Personal, Indoor and Mobile Radio Communications (PIMRC), pages 1-7. IEEE. 
Thubert, P. (2012). Objective function zero for the routing protocol for low-power and lossy networks (rpl). Technical report.

Wang, D., Li, W., and Wang, P. (2018). Measuring twofactor authentication schemes for real-time data access in industrial wireless sensor networks. IEEE Transactions on Industrial Informatics.

Wang, J. and Chalhoub, G. (2019). Mobility support enhancement for $\mathrm{rpl}$ with multiple sinks. Annals of Telecommunications, pages 1-14.

Wang, Z., Zhang, L., Zheng, Z., and Wang, J. (2016). An optimized rpl protocol for wireless sensor networks. In Parallel and Distributed Systems (ICPADS), 2016 IEEE 22nd International Conference on, pages 294299. IEEE.

Winter, T., Thubert, P., Brandt, A., Hui, J., Kelsey, R., Levis, P., Pister, K., Struik, R., Vasseur, J.-P., and Alexander, R. (2012). Rpl: Ipv6 routing protocol for low-power and lossy networks. Technical report.

Zeinab, K. A. M. and Elmustafa, S. A. A. (2017). Internet of things applications, challenges and related future technologies. World Scientific News, 2(67):126-148. 